The Scientist-Practitioner on the Front Line

Pragmatic Case Studies in Psychotherapy, http://pcsp.libraries.rutgers.edu

Volume 1, Module 3, Article 4, pp. 1-4, 10-21-2005 [copyright by author]

Response to Commentaries on The Case of "CG:" Balancing Supportive and Insight-Oriented Psychodynamic Psychotherapy with a Client Undergoing Intense Life Stress

\title{
The Roles of the EBPP Model and of Bio-Medical Approaches in Psychodynamic Therapy
}

\author{
KAREN RIGGS SKEAN ${ }^{\mathrm{a}, \mathrm{b}}$ \\ ${ }^{\text {a }}$ Graduate School of Applied and Professional Psychology, Rutgers - The State University of New Jersey \\ Correspondence concerning this article should be addressed to Karen Riggs Skean, Rutgers University, Graduate \\ School of Applied and Professional Psychology, 152 Frelinghuysen Road, Piscataway, NJ 08854. \\ Email: kskean@aol.com
}

\begin{abstract}
Goodheart (2005) and Miller (2005) provide important additional dimensions to psychodynamic case studies like mine (Skean, 2005a). Goodheart argues persuasively for making such case studies more publicly accountable by more clearly connecting them to the principles of APA's recently finalized Evidence-Based Practice in Psychology (EBPP) model. This includes the use of more formalized and standardized measures of therapy process and outcome, including the DSM categories of the American Psychiatric Association, and the use of explicit strategies for minimizing the distortions of cognitive biases and improving judgment. Miller, on the other hand, critiques the use of the DSM and other bio-medical approaches, such as psychopharmacology. I respond to both sets of important ideas, delineating areas of agreement and disagreement with the aim of encouraging other psychodynamic practitioners to address them openly and constructively.
\end{abstract}

Key words: psychodynamic therapy; Evidence-Based Practice in Psychology (EBPP); Disagnostic Statistical Manual (DSM)

I welcome the informative commentaries of both Carol Goodheart (2005) and Ronald Miller (2005) about my case study (Skean, 2005a). Goodheart's commentary brings to bear her cutting edge work and public policy perspective on the Evidence-Based Practice in Psychology (EBPP) model. Her examination of the case in terms of the clear mandate of EBPP to pay attention to best available research, clinical expertise, and patient characteristics and values is timely indeed. Her careful explication of the tenets of EBPP as applied to the case should set a helpful standard for future cases in this journal. It is particularly useful to see these criteria applied to a case that uses a psychodynamic framework, as practitioners of that orientation have often perceived the arena of evidence-based practice to be dominated by manualized protocols and cognitive-behavioral approaches, and to be of little positive relevance to their work. While acknowledging that fundamental philosophical differences are still being debated between 
The Scientist-Practitioner on the Front Line

K.R. Skean

Pragmatic Case Studies in Psychotherapy, http://pcsp.libraries.rutgers.edu

Volume 1, Module 3, Article 4, pp. 1-4, 10-21-2005 [copyright by author]

proponents of a medical model of psychotherapy based on testing specific components in controlled studies and a more contextual model of psychotherapy, Goodheart demonstrates that there are many points of applicability for evidence-based approaches to a case that is neither manualized nor cognitive behavioral.

Miller comments on a missed opportunity for collaborative input on the case study from other professionals involved in the case. At the time of writing this now, I would not have had access to those inputs, as this was from an earlier point in my career. Yet his point did make me ponder our tendency to think of case studies as a one-person (or, including the client, twoperson) enterprise. The intimacy and privacy of the therapeutic setting lends itself to the development of a narrative that organizes and explicates what happened in that bi-personal field. The idea of opening this setting up, especially prospectively, to other minds and voices, would seem to demand a new and rich model. As responsible clinicians, we are often involved in incorporating other points of view into our work to guard against blind spots - supervision groups, consultations - but these too take place in intimate and private settings, and we do not often think of a model of more public case presentation (like the PCSP journal) that would include those inputs in any systematic way.

Goodheart also speaks from her work on the EBPP model about the need for minimizing the distortions of cognitive biases and improving judgment. Her emphasis is on more formalized measures, both during the evaluation and in assessing outcome, which would clearly have increased the knowledge value of the current case.

Miller comments on my lack of attention to some aspects of my client's real world and the possibility that the treatment underacknowledged the potential injustices of his treatment at the hands of his department. The favoring of intrapsychic over social and political realities has historically been a weakness of the psychodynamic tradition, and one which has led at times to "blaming the victim" and to an underestimation of real world injustices, abuses of power, and the dysfunction of larger institutions with whom the client interacts. The feminist critique (Luepnitz, 1988) and now the emphasis on cultural competency (Perez-Foster, Moskowitz, \& Javier, 1996) in psychoanalysis has drawn the attention of the psychodynamic community to this blinder. I think, given the strength that developed in the therapeutic alliance, my client's experience of me was one of being "on his side." However, that could have been stronger if made more explicit: there is a difference between being on the client's side in a generic supportive sense and being on the client's side in an informed and theorized way that allows creation of a new perspective on an important part of the client's lived world. I have become more aware of the importance of this (Skean, 2005b) and believe it can be incorporated into an overall psychodynamic guiding conception. I have found that incorporating into therapy an exploration with the client of the dynamics of his or her workplace, and how those dynamics or "social defenses" might be colliding with the client's own current circumstances and enduring vulnerabilities, is helpful and depathologizing. Were I to see CG now, I would be more alert to this.

Miller carries forward his sensitivity to the dynamics of power and authority in psychotherapy in his critique of DSM-based thinking and the too-automatic referral for 
medication evaluation. Miller finds the use of the DSM-IV diagnosis (American Psychiatric Association, 2000) and the medication consultation an unwelcome and unnecessary intrusion in the case, reflecting an offering to the gods of the medical model and not truly related to the guiding conception. There is justice in that. I do find, in re-reading my case, that the section on the DSM diagnosis is, by comparison, drier, more stilted and less connected to the lived world of the client. Yet, it reflects practice in a world where few of us are free to march only to our own drummer. Third-party payers, agency reviewers, even clients who have self-diagnosed on the Internet, pull us into this other conversation. Even if that language is not our own, we have to be able to speak it. I have found that thinking about aspects of cases through the lens of that different paradigm has been useful and has alerted me to missed aspects in my evaluations. From her perspective, Goodheart does not seem troubled by the inclusion of the DSM, and indeed seems to find it consistent with EBPP, observing that our use of "operationalized guideposts that cross theoretical orientations" can provide one of those checks that can help to reduce bias and error.

While I find many points of agreement with Miller's basic critique of DSM, we have more differences regarding the usefulness of medication. It is true that this most recent era in mental health treatment has been excessively dominated by a reliance on psychotropic medication, often accompanied by a devaluation of psychological methods. Still, I have seen instances where medication truly has done something that therapy had not been able to, when the client says, "I should have done this 10 years ago! My father should have done this 30 years ago!" While results vary widely, I would not feel justified in denying the client the opportunity to consider this choice, when there is a possibility it might provide some measure of relief from suffering. We are in an era of new respect for mind/body interaction and for the importance of the body in psychotherapy. In this post-Cartesian world, we do not have to make an either/or choice. Our tools are still crude, and yes, the pharmaceutical side is driven by a corporate, profit-oriented machine. But the bigger principle is that of including clients collaboratively in decisions about treatment options and adjuncts, whether they be medication, cognitive-behavior therapy, psychodynamic therapy, yoga, biofeedback, or an exercise program. Candor about the risks and benefits of these, as understood by the practitioner, need not be viewed as a confusing undercutting of the therapeutic relationship, but as respectful partnering, the collaboration between clinician and client that Goodheart speaks of as a central tenet of EBPP.

Now was this the spirit in which I handled the medication decision with CG? No, it was more the knee-jerk reaction of the young clinician frightened by a client's deteriorating functioning and following accepted practice, as Miller astutely surmises. Still, in the context of the relationship, I think CG was able to read the episode as a reflection of my concern for him and my wish that we find a way to reduce his suffering. I was fortunate, for purposes of the present case study, that medication proved not to be an option. It would certainly have clouded the discussion of the benefits of treatment, and made it very easy to attribute improvements to medication rather than to the therapeutic relationship and/or to theory-informed psychological intervention. This is one of the dilemmas associated with case studies of ordinary clinical practice. The pragmatism of clinicians, of which Goodheart speaks, leads us to combine interventions in a way that makes elucidating the comparative power of particular components 
difficult. If she is correct that the sea change in the wider political context is soon to raise the bar on standards of measurement and evaluation, perhaps this will change.

\section{REFERENCES}

American Psychiatric Association (2000). Diagnostic and statistical manual of mental disorders: DSM-IV-TR (Text Revision). Washington, D.C.: Author.

Goodheart, C.D. (2005). Placing psychotherapy case studies with the framework of the APA Evidence-Based Practice in Psychology (EBPP) model. Pragmatic Case Studies in Psychotherapy, Psychotherapy [Online], Vol. 1(3), Article 2. Available: http://hdl.rutgers.edu/1782.1/pcsp_journal

Luepnitz, D. (1988). The family interpreted: Feminist theory in clinical practice. New York: Basic Books.

Miller, R.B. (2005). Adding supporting evidence and eliminating extraneous bio-medical constructs from the psychodynamic case study. Pragmatic Case Studies in Psychotherapy, Psychotherapy [Online], Vol. 1(3), Article 3. Available: http://hdl.rutgers.edu/1782.1/pcsp_journal

Perez-Foster, R., Moskowitz, M., \& Javier, R., eds. (1996). Reaching across boundaries of culture and class: Widening the scope of psychotherapy. Northvale, NJ: Jason Aronson.

Skean, K.R. (2005a). The case of "CG:” Balancing supportive and insight-oriented psychodynamic psychotherapy with a client undergoing intense life stress. Pragmatic Case Studies in Psychotherapy, Psychotherapy [Online], Vol. 1(3), Article 1. Available: http://hdl.rutgers.edu/1782.1/pcsp_journal

Skean, K. R. (2005b) Organizing the individual psychotherapist. New Jersey Psychologist, 55, 28-30. 